\title{
A halogen bond-donor amino acid for organocatalysis in water $\dagger$
}

\author{
Greta Bergamaschi, ${ }^{* a}$ Luisa Lascialfari, $\neq^{a}$ Andrea Pizzi, a Maria Isabel Martinez Espinoza, ${ }^{a}$ Nicola Demitri, ${ }^{b}$ \\ Alessandro Gori*c and Pierangelo Metrangolo ${ }^{a}$ Alberto Milani,
}

Herein we report a halogen bond-donor amino acid, 4-iodotetrafluorophenylalanine, which behaves as a catalyst for the aqueous synthesis of bis-(heterocyclic)methanes. We also provide experimental evidence that halogen bonding is a plausible explanation for the observed catalytic effect.

A halogen bond $(\mathrm{XB})$ is the noncovalent interaction between an electrophilic halogen atom acting as a Lewis acid (XB-donor) and a nucleophilic species behaving as a Lewis base (XB-acceptor). ${ }^{1}$ The XB has been exploited for many applications, with a particular emphasis on crystal engineering and XB-driven assembly of supramolecular structures. ${ }^{2}$ The use of $\mathrm{XB}$ in organocatalysis is also rapidly growing and, in this frame, it is remarkable that $\mathrm{XB}$ was recently rationalized as the origin of molecular iodine catalytic activity in certain organic transformations (e.g., Michael addition, Friedel-Crafts reaction, etc.). ${ }^{3}$ Yet, XB-based catalysis remains a largely unexplored topic.

Recent examples reported on the use of positively charged or neutral XB-donor catalysts for the electrophilic activation of substrates in a plethora of organic transformations. ${ }^{4,5}$ However, to the best of our knowledge, catalyst application was limited to reactions in organic solvents. This is probably due to the scarce aqueous solubility of common XB-donor scaffolds, typically containing iodopolyfluoroaromatic moieties. The development of more water soluble catalysts may therefore open up new perspectives in the field of XB-driven organocatalysis. In this

\footnotetext{
${ }^{a}$ Department of Chemistry, Materials, and Chemical Engineering "Giulio Natta", Politecnico di Milano, Via L. Mancinelli 7, 20131 Milan, Italy.

E-mail: greta.bergamaschi@polimi.it

${ }^{b}$ Elettra-Sincrotrone Trieste, S.S. 14 Km 163.5 in Area Science Park, 34149

Basovizza, Trieste, Italy

${ }^{c}$ Istituto di Chimica del Riconoscimento Molecolare (ICRM-CNR),

via Mario Bianco 9, 20131 Milano, Italy. E-mail: alessandro.gori@icrm.cnr.it

$\dagger$ Electronic supplementary information (ESI) available: Experimental details and supplemental figures, table and CIF file. CCDC 1840351 and 1841117. For ESI and crystallographic data in CIF or other electronic format see DOI: 10.1039/ c8cc06010j

‡ L. F. presently works at Brachi Company, Prato, Italy.
}

context, we envisioned that by introducing the 4-iodo-2,3,5,6tetrafluorophenyl moiety in an amino acid scaffold, we would have preserved its XB-donor properties while imparting sufficient water solubility to allow catalysis in aqueous media. Consequently, we focused our attention on the unnatural XB-donor amino acid 2,3,5,6-tetrafluoro-4-iodophenylalanine $\mathbf{F}\left(\mathbf{F}_{\mathbf{4}} \mathbf{I}\right)$ (Fig. 1a), for which the synthesis of the corresponding Fmoc-protected analogue was previously described. ${ }^{6}$ In our hands, obtaining large amounts of the latter by means of the reported multistep procedure proved to be a laborious and timeconsuming task. Therefore, we designed a new and straightforward synthetic strategy that provided us with the unprotected $\mathbf{F}\left(\mathbf{F}_{\mathbf{4}} \mathbf{I}\right)$ amino acid, as a trifluoroacetate (TFA) salt, on a gram-scale and good yields ( $32 \%$ overall). To this end, it was key for the new procedure to consistently reduce the number of synthetic steps down to six (see Scheme S1, ESI $\dagger$ ).

The computed molecular electrostatic potential (MEP) of $\mathbf{F}\left(\mathbf{F}_{4} \mathbf{I}\right)$ shows the existence of a relevant $\sigma$-hole (Fig. $1 \mathrm{~b}$ ) with a MEP value of $128 \mathrm{~kJ} \mathrm{~mol}^{-1}$ along the extension of the $\mathrm{C}-\mathrm{I}$ bond, which is comparable to values reported in the literature. ${ }^{7}$ This was also confirmed by the single-crystal X-ray structure of $\mathbf{F}\left(\mathbf{F}_{\mathbf{4}} \mathbf{I}\right)$, after it crystallized from a 1:1 water/acetone mixture in a chiral monoclinic unit cell ( $P 2_{1}$ space group), with three amino acid molecules in the asymmetric unit. Its crystal structure clearly<smiles>NC(Cc1c(F)c(F)c(I)c(F)c1F)C(=O)O</smiles>

$F\left(F_{4} I\right)$

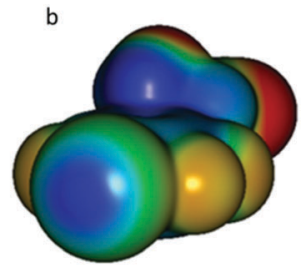

Fig. 1 (a) Chemical structure of $\mathbf{F}\left(F_{4} \mathbf{l}\right)$; (b) plot of the MEP computed by means of DFT calculations on the 0.001 a.u. contour of the isodensity surface (see the ESI $\uparrow 8$ for computational details). Colour ranges adopted in the map are (in $\mathrm{kcal} \mathrm{mol}^{-1}$ ): red, lower than -18.8; yellow -6.3; green 0.0 ; light blue +6.3 ; blue, larger than +25.1 . 


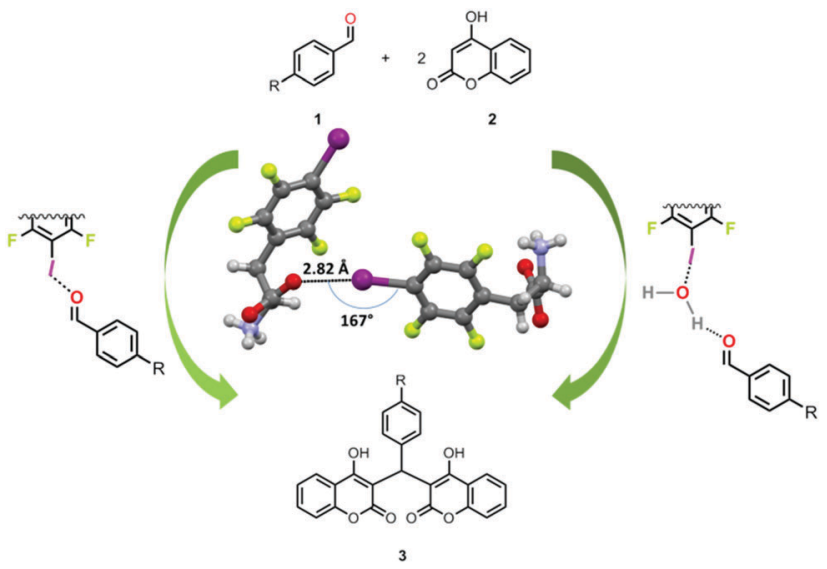

Fig. 2 Possible carbonyl-catalyst interactions. For the sake of simplicity only interactions involving aldehydes are depicted, but those involving 4-hydroxycoumarin are not excluded: (left) formation of a genuine XB between carbonyl oxygen and polarized iodine of $\mathbf{F}\left(F_{4} \mathbf{l}\right)$; (right) water mediated $\mathrm{XB}$. Inset: Partial crystal structure of $\mathbf{F}\left(\mathrm{F}_{\mathbf{4}} \mathbf{I}\right)$ showing the halogen bond involving the iodine atom and the carbonyl oxygen of a nearby amino acid molecule (colour code: carbon: grey; iodine: purple; fluorine: light green; oxygen: red; hydrogen: white)

shows the ability of $\mathbf{F}\left(\mathbf{F}_{\mathbf{4}} \mathbf{I}\right)$ to form a strong halogen bond with a

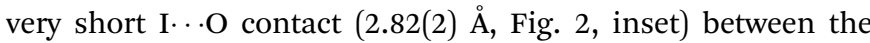
iodine atom and the carbonyl oxygen of an adjacent molecule (C-I $\cdots$ O angle: $\left.167.0(5)^{\circ}\right)$. XB occurs almost orthogonally to the hydrogen bonding involving the same carbonyl oxygen with the $\mathrm{NH}_{3}{ }^{+}$group of an adjacent molecule $(\mathrm{I} \cdots \mathrm{O} \cdots \mathrm{N}$ angle of 106.7(7) ${ }^{\circ}$, see the ESI $\dagger$ for details).

Given the outstanding XB-donor properties of $\mathbf{F}\left(\mathbf{F}_{\mathbf{4}} \mathbf{I}\right)$, we moved to explore its possible XB-mediated catalytic applications in water taking as a model reaction the synthesis of bis-(heterocyclic)methanes, using benzaldehyde and 4-hydroxycoumarin as substrates. ${ }^{8}$ Previous literature reported this reaction to proceed spontaneously at $100{ }^{\circ} \mathrm{C}$ in 5 hours. ${ }^{9}$ However, we could immediately assess that the addition of $0.2 \% \mathbf{F}\left(\mathbf{F}_{4} \mathbf{I}\right)$ in the reaction mixture considerably increased the rate of product formation ( $>97 \%$ in 20 minutes, Table S1 (ESI $\dagger$ ), entry 1). Remarkably, the presence of $0.2 \% \mathbf{F}\left(\mathbf{F}_{4} \mathbf{I}\right)$ significantly improved the product formation also at a lower temperature $\left(97 \%\right.$ in $\left.1 \mathrm{~h}, 60{ }^{\circ} \mathrm{C}\right)$.

In order to consolidate our initial hypothesis on the possible role of $\mathrm{XB}$ in promoting a catalytic interaction with the substrates, we screened differently polarized halogenated phenylalanines to assess whether weakening or eliminating the XB-donor properties of the catalyst may alter the reaction kinetics. To this end, we used L-phenylalanine (Phe), pentafluoro-L-phenylalanine ( $\mathrm{F}_{5}$-Phe), and 4-bromo-L-phenylalanine (Br-Phe) for their absent/poor XB-donor propensity, respectively, while 4-iodo-L-phenylalanine (I-Phe) was expected to maintain a certain catalytic activity despite it lacking the polyfluorinated aromatic substitution that exacerbates the iodine atom polarizability. Finally, tyrosine (Tyr) was used as a hydrogen bond (HB)-donor catalyst to compare $\mathrm{HB}$ with $\mathrm{XB}$ in the same experimental set-up. We set conditions for comparison at $60{ }^{\circ} \mathrm{C}$ and evaluated catalyst efficiencies by calculating initial rates (slopes) in the linear traits of reaction curves. With respect to the spontaneous

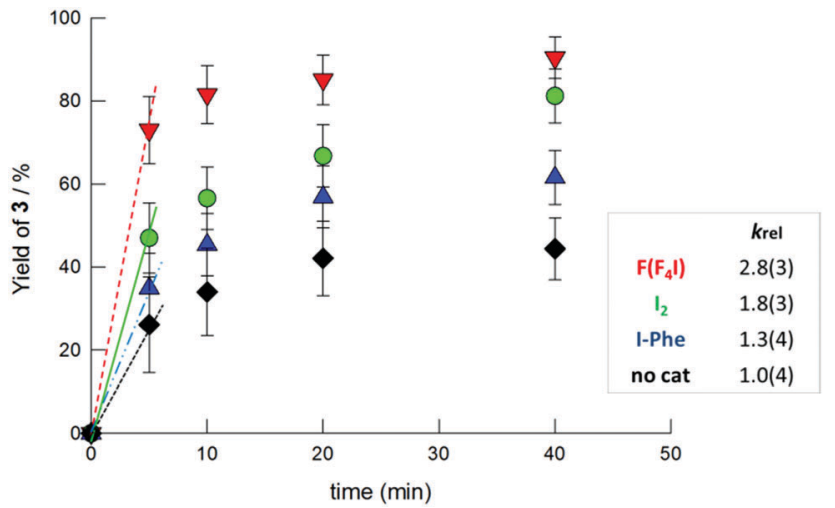

Fig. 3 Conversion vs. time curves for the benchmark reaction performed at $60{ }^{\circ} \mathrm{C}$ and linear fits for the determination of initial slopes. $k_{\text {rel }}$ values are relative to the non-catalyzed reactions.

reaction, no significant acceleration rate was detected in the presence of Phe, $\mathrm{F}_{5}$-Phe, and Br-Phe. Consistently, the addition of I-Phe, iodine, and $\mathbf{F}\left(\mathbf{F}_{\mathbf{4}} \mathbf{I}\right)$ considerably increased the rate of product formation, instead. By linear regression of the initial slope of conversion versus time, we could estimate that the $\mathbf{F}\left(\mathbf{F}_{4} \mathbf{I}\right)$ relative reaction rate constant $\left(k_{\text {rel }}\right)$ is about 3-fold higher than that of the uncatalyzed reaction (Fig. 3).

Similar results were obtained in the presence of the amineprotected derivative Fmoc-F( $\left.\mathbf{F}_{4} \mathbf{I}\right)$, thus excluding the possible involvement of the zwitterionic ammonium moiety in the catalysis (Table S2 and Fig. S2, ESI $\dagger$ ). It should be noted that tyrosine, despite being a good hydrogen bond-donor, only poorly activates the substrate, suggesting that halogen bonding is more effective in water than hydrogen bonding because of its stronger hydrophobicity. ${ }^{10}$ Furthermore, the fact that TFA salts of Br-Phe, Tyr, and $\mathbf{F}\left(\mathbf{F}_{4} \mathbf{I}\right)$ show quite different activities allows the exclusion of a major role of acid traces in the catalysis. Overall, these results support a plausible involvement of XB in the reaction activation mechanism.

We then additionally tested a number of aromatic aldehydes differing in the presence of electron-withdrawing (EWG) and electron-donating (EDG) groups at the para position of the phenyl ring (Table S3, ESI $\dagger$ ). As expected, the nature of the aromatic substituents on aldehyde substrates significantly impacted these catalyzed reactions, with more electrophilic aldehydes being the most reactive. Interestingly, this trend was not observed in the absence of the catalyst, i.e., the spontaneous reaction (see Table S3, ESI $\dagger$ ).

In order to further establish the occurrence of an interaction between the substrates and the catalyst, we moved to spectroscopic analyses (NMR and ATR-FTIR), DSC, and single-crystal X-ray diffraction experiments. ${ }^{19} \mathrm{~F}-\mathrm{NMR}$ spectroscopy experiments demonstrated that under the reaction conditions, $\mathbf{F}\left(\mathbf{F}_{\mathbf{4}} \mathbf{I}\right)$ fluorine atoms in the ortho positions to the C-I fragment undergo a $0.85 \mathrm{ppm}$ up-field shift (Fig. S1, ESI + ), which is typical of the C-I fragment involvement in XB. ${ }^{1 a}$ Unfortunately, all attempts to obtain 1:1 $\mathbf{F}\left(\mathbf{F}_{4} \mathbf{I}\right)$ co-crystals with the starting substrates were unsuccessful. However, this was not totally unexpected, since dimeric halogen-bonded complexes have usually low melting 


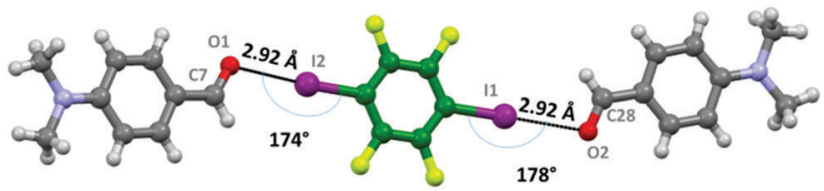

Fig. $4 \mathrm{XBs}$ in the co-crystal formed between 1,4-DITFB and 1b. The halogen bond occurs orthogonally to the carbonyl oxygen with an angle $12 \ldots \mathrm{O} 1 \cdots \mathrm{C} 7$ of $113.6(2)^{\circ}$ and $11 \cdots \mathrm{O} 2 \ldots \mathrm{C} 28$ of $107.6(3)^{\circ}$. Carbon: grey and green; iodine: purple; fluorine: yellow; oxygen: red; hydrogen: light grey.

points and the formation of stable co-crystals may prove challenging. ${ }^{11}$ For this reason, we turned our attention to the co-crystallization of $N, N$-dimethyl amino benzaldehyde (1b) with 1,4-diiodotetrafluorobenzene (1,4-DITFB), a very wellknown and strong ditopic XB-donor, as a model of the iodine-carbonyl interaction. Single crystals were obtained from a $1: 1$ chloroform solution after evaporating the solvent in three days. The asymmetric unit consists of two aldehyde molecules and two 1,4-DITFB units. Both XB-donor modules form XBs with the carbonyl oxygen atoms of $\mathbf{1} \mathbf{b}$. The C-I $\cdots$ O synthon drives the self-assembly of a supramolecular trimer (Fig. 4); both XB-donor modules form short XBs with the carbonyl oxygens of 1b (Fig. S11, ESI $\dagger$ ). It is worth underlining that aldehyde $\mathbf{1 b}$ is the less activated substrate towards the condensation with 4-hydroxycoumarin. Here, it is easy to appreciate the key role of $\mathrm{XB}$ in lowering the electron density on the aldehyde carbon, hence activating also a substrate that is very less prone to react without a catalyst (see Table S3, ESI $\dagger$ ). It is also noteworthy that the iodine atoms of 1,4-DITFB are actually less pronounced $\mathrm{XB}$-donor groups compared to the single iodine atom of iodopentafluorobenzene. ${ }^{1 a}$

In order to sample the formation of new XB-mediated coordinative species on a bulk co-crystal population, we performed differential scanning calorimetry (DSC) experiments using either iodopentafluorobenzene (IPFB) or 1,4-DITFB as model XB-donors and aldehydes $\mathbf{1 a}$ and $\mathbf{b}$ as acceptors. New single crystallization and melting peaks appeared in their 1:1 mixtures (Table S4, Fig. S3 and S4, ESI $\dagger$ ), suggesting the occurrence of XB. Additional indications of XB were suggested by ATR-FTIR experiments on different amorphous solids obtained by mixing aldehydes 1b-1d and 1,4-DITFB in the 1:1 molar ratio (see the ESI $\dagger$ ). In particular, a red-shift of the C-I stretching vibration bands of 1,4-DITFB occurred in the spectrum of the adduct with the XB-acceptor (see Fig. S5-S7, ESI $\dagger$ ). This peculiar fingerprint was previously attributed to the lengthening and the weakening of the $\mathrm{C}-\mathrm{I}$ bond involved in the XB formation with the carbonyl oxygen of $\mathbf{1 b} .^{11,12}$ Moreover, the carbonyl broad band of aldehydes undergoes a red-shift in the FT-IR spectrum of the co-crystal. ${ }^{11 c}$

Overall, all the obtained experimental evidence jointly and consistently points towards the potential involvement of XB in the activation of the condensation reaction. The observed catalytic effect may result from the direct activation of the carbonyl group through a genuine XB between the carbonyl oxygen and the polarized iodine atom of $\mathbf{F}\left(\mathbf{F}_{4} \mathbf{I}\right)$, as the reported crystal structures suggest. Alternatively, it may result from a very interesting effect of the water environment through, e.g., a water-bridged $\mathrm{XB}$ mechanism involved in the aldehyde activation (Fig. 2, right). ${ }^{13}$ A concurrent contribution of both mechanisms is also likely to be involved.

In conclusion, in the present work we explored the possibility to perform XB-promoted catalysis in water by exploiting an XB-donor amino acid $\mathbf{F}\left(\mathbf{F}_{4} \mathbf{I}\right)$ that combines excellent XB-donor properties with good water solubility. To the best of our knowledge, this is the first case of an XB-catalyzed reaction in water involving an amino acid, paving the way towards a new broader and more sustainable perspective in XB-promoted catalysis applications. Moreover, the amino acid $\mathbf{F}\left(\mathbf{F}_{\mathbf{4}} \mathbf{I}\right)$ is a new and powerful building block for the straightforward incorporation of the catalytic tetrafluoroiodo moiety into higher complexity structures, such as peptide-based scaffolds.

This work was partially supported by the European Research Council (ERC) through the grant FOLDHALO (no. 307108). CARIPLO Foundation is also gratefully acknowledged for partial financial support (grant no. 2014-0746 and 2016-0472).

\section{Conflicts of interest}

There are no conflicts of interest to declare.

\section{Notes and references}

1 (a) G. Cavallo, P. Metrangolo, R. Milani, T. Pilati, A. Priimagi, G. Resnati and G. Terraneo, Chem. Rev., 2016, 116, 2478; (b) A. Brown and P. D. Beer, Chem. Commun., 2016, 52, 8645; (c) T. M. Beale, M. G. Chudzinski, M. G. Sarwar and M. S. Taylor, Chem. Soc. Rev., 2012, 42, 1667; (d) M. Erdelyi, Chem. Soc. Rev., 2012, 41, 3547; (e) G. R. Desiraju, P. S. Ho, L. Kloo, A. C. Legon, R. Marquardt, P. Metrangolo, P. Politzer, G. Resnati and K. Rissanen, Pure Appl. Chem., 2013, 85, 1711; $(f)$ A. V. Jentzsch and S. Matile, Top. Curr. Chem., 2015, 358.

2 (a) L. C. Gilday, S. W. Robinson, T. A. Barendt, M. J. Langton, B. R. Mullaney and P. D. Beer, Chem. Rev., 2015, 115, 7118; (b) A. V. Jentzsch, A. Hennig, J. Mareda and S. Matile, Acc. Chem. Res., 2013, 46, 2791; (c) C. A. Gunawardana, M. Daković and C. B. Aakeröy, Chem. Commun., 2018, 54, 606.

3 (a) D. Heiden, S. Bozkus, M. Klussmann and M. Breugst, J. Org. Chem., 2017, 82, 4037; (b) M. Breugst, E. Detmar and D. Heiden, ACS Catal., 2016, 6, 3203.

4 (a) S. H. Jungbauer, S. M. Walter, S. Schindler, L. Rout, F. Kniep and S. M. Huber, Chem. Commun., 2014, 50, 6281; (b) F. Kniep, S. H. Jungbauer, Q. Zhang, S. M. Walter, S. Schindler, I. Schnapperelle, E. Herdtweck and S. M. Huber, Angew. Chem., Int. Ed., 2013, 52, 7028; (c) S. H. Jungbauer and S. M. Huber, J. Am. Chem. Soc., 2015, 137, 12110; (d) D. Bulfield and S. M. Huber, Chem. - Eur. J., 2016, 22, 14434; (e) A. Bruckmann, M. A. Pena and C. Bolm, Synlett, 2008, 900; $(f)$ Y. Takeda, D. Hisakuni, C. H. Lin and S. Minakata, Org. Lett., 2015, 17, 318.

5 (a) A. Dreger, E. Engelage, B. Mallick, P. D. Beer and S. M. Huber, Chem. Commun., 2018, 54, 4013; (b) J. P. Gliese, S. H. Jungbauer and S. M. Huber, Chem. Commun., 2017, 53, 12052; (c) M. Saito, Y. Kobayashi, S. Tsuzuki and Y. Takemoto, Angew. Chem., Int. Ed., 2017, 56, 7653; (d) W. He, Y. C. Ge and C. H. Tan, Org. Lett., 2014, 16, 3244; $(e)$ N. Tsuji, Y. Kobayashi and Y. Takemoto, Chem. Commun., 2014, 50, 13691; $(f)$ Y. Kobayashi, Y. Nakatsuji, S. Li, S. Tsuzuki and Y. Takemoto, Angew. Chem., Int. Ed., 2018, 57, 3646; $(g)$ Y. Cheung Chan and Y. Y. Yeung, Angew. Chem., Int. Ed., 2018, 57, 3483; (h) C. Huo, M. Wu, F. Chen, X. Jia, Y. Yuan and H. Xie, Chem. Commun., 2015, 51, 4708; (i) I. Kazi, S. Guha and G. Sekar, Org. Lett., 2017, 19, 1244.

6 L. Qin, C. Sheridan and J. Gao, Org. Lett., 2012, 14, 528. 
7 (a) P. Politzer, P. Lane, M. C. Concha, Y. Ma and J. S. Murray, J. Mol. Model., 2007, 13, 305; (b) P. Politzer, J. S. Murray and T. Clark, Phys. Chem. Chem. Phys., 2010, 12, 7748; (c) C. B. Aakeröy, M. Baldrighi, J. Desper, P. Metrangolo and G. Resnati, Chem. - Eur. J., 2013, 19, 16240.

8 (a) M. Seddighi, F. Shirinin and M. Mamaghani, RSC Adv., 2013, 3, 24046; (b) M. Seddighi, F. Shirini and M. Mamaghani, RSC Adv., 2013, 3, 24046; (c) B. Dam, S. Nandi and A. K. Pal, Tetrahedron Lett., 2014, 55, 5236.

9 S. Yaragorla, A. Pareek and R. Dada, Tetrahedron Lett., 2015, 56, 4770.

10 C. C. Robertson, J. S. Wright, E. J. Carrington, N. Perutz, C. A. Hunter and L. Brammer, Chem. Sci., 2017, 8, 5392.
11 (a) V. Vasylyeva, L. Catalano, C. Nervi, R. Gobetto, P. Metrangolo and G. Resnati, CrystEngComm, 2016, 18, 2247; (b) N. F. Cheethaman and A. D. E. Pullin, Chem. Commun., 1967, 233; (c) V. Vasylyeva, S. K. Nayak, G. Terraneo, G. Cavallo, P. Metrangolo and G. Resnati, CrystEngComm, 2014, 16, 8102.

12 (a) X. Ding, M. J. Tuikka, P. Hirva, V. Y. Kukushkin, A. S. Novikovd and M. Haukka, CrystEngComm, 2016, 18, 1987; (b) C. Laurence, J. Graton, M. Berthelot and M. J. El-Ghomari, Chem. - Eur. J., 2011, 17, 10431.

13 L. Mendez, G. Henriquez, S. Sirimulla and M. Narayan, Molecules, 2017, 22, 1397; S. Z. Borozan and S. D. Stojanovi, Comput. Biol. Chem., 2013, 47, 231. 